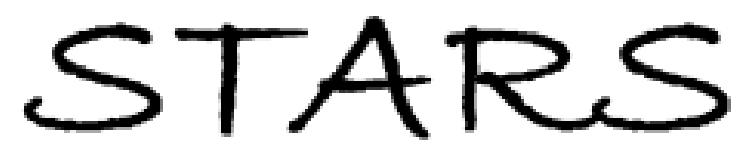

University of Central Florida

STARS

$1-1-2009$

\title{
Solution processed large area field effect transistors from dielectrophoreticly aligned arrays of carbon nanotubes
}

\author{
Paul Stokes \\ University of Central Florida \\ Eliot Sibar \\ University of Central Florida \\ Yashira M. Zayas \\ University of Central Florida \\ Saiful I. Khondaker \\ University of Central Florida
}

Find similar works at: https://stars.library.ucf.edu/facultybib2000

University of Central Florida Libraries http://library.ucf.edu

This Article is brought to you for free and open access by the Faculty Bibliography at STARS. It has been accepted for inclusion in Faculty Bibliography 2000s by an authorized administrator of STARS. For more information, please contactSTARS@ucf.edu.

\section{Recommended Citation}

Stokes, Paul; Sibar, Eliot; Zayas, Yashira M.; and Khondaker, Saiful I., "Solution processed large area field effect transistors from dielectrophoreticly aligned arrays of carbon nanotubes" (2009). Faculty Bibliography 2000s. 2186.

https://stars.library.ucf.edu/facultybib2000/2186

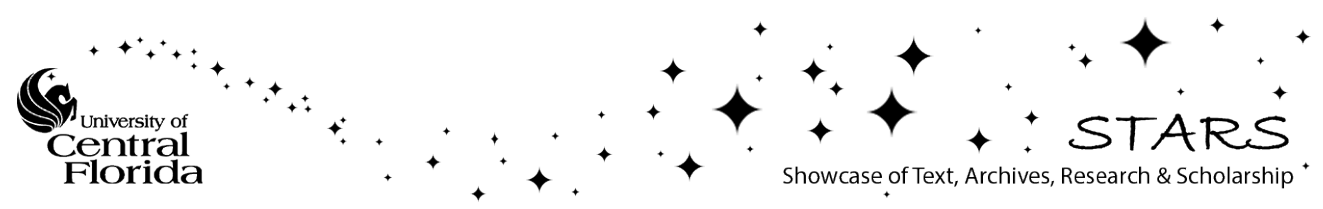




\section{Solution processed large area field effect transistors from dielectrophoreticly aligned arrays of carbon nanotubes}

Cite as: Appl. Phys. Lett. 94, 113104 (2009); https://doi.org/10.1063/1.3100197

Submitted: 02 December 2008 . Accepted: 25 February 2009 . Published Online: 16 March 2009

Paul Stokes, Eliot Silbar, Yashira M. Zayas, and Saiful I. Khondaker

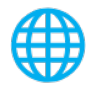

\section{ARTICLES YOU MAY BE INTERESTED IN}

High quality solution processed carbon nanotube transistors assembled by dielectrophoresis

Applied Physics Letters 96, 083110 (2010); https://doi.org/10.1063/1.3327521

Directed assembly of solution processed single-walled carbon nanotubes via dielectrophoresis: From aligned array to individual nanotube devices Journal of Vacuum Science \& Technology B 28, C6B7 (2010); https:// doi.org/10.1116/1.3501347

High-frequency performance of scaled carbon nanotube array field-effect transistors Applied Physics Letters 101, 053123 (2012); https://doi.org/10.1063/1.4742325

\section{Applied Physics Letters}

Mid-IR and THz frequency combs special collection 


\title{
Solution processed large area field effect transistors from dielectrophoreticly aligned arrays of carbon nanotubes
}

\author{
Paul Stokes, Eliot Silbar, Yashira M. Zayas, ${ }^{a}$ and Saiful I. Khondaker ${ }^{\text {b) }}$ \\ Nanoscience Technology Center and Department of Physics, University of Central Florida, 12424 Research \\ Parkway, Orlando, Florida 32826, USA
}

(Received 2 December 2008; accepted 25 February 2009; published online 16 March 2009)

\begin{abstract}
We demonstrate solution processable large area field effect transistors (FETs) from aligned arrays of carbon nanotubes (CNTs). Commercially available, surfactant free CNTs suspended in aqueous solution were aligned between source and drain electrodes using ac dielectrophoresis technique. After removing the metallic nanotubes using electrical breakdown, the devices displayed $p$-type behavior with on-off ratios up to $\sim 2 \times 10^{4}$. The measured field effect mobilities are as high as $123 \mathrm{~cm}^{2} / \mathrm{V} \mathrm{s}$, which is three orders of magnitude higher than typical solution processed organic FET devices. (C) 2009 American Institute of Physics. [DOI: 10.1063/1.3100197]
\end{abstract}

Solution processed electronic devices have attracted tremendous attention because of their ease of processablity, low cost of fabrication, and ability to cover large areas. These devices may be useful for applications such as flexible displays, sensor sheets, radio frequency identification tags, and photovoltaics. ${ }^{1,2}$ A significant amount of effort has been dedicated to improve device performance of solution processed organic field effect transistors (FETs). However, typical field effect mobilities for these devices are usually on the order of $\sim 0.1 \mathrm{~cm}^{2} / \mathrm{V} \mathrm{s}$ and can very rarely reach $\sim 1.0 \mathrm{~cm}^{2} / \mathrm{V} \mathrm{s} .{ }^{1-3}$ In addition, the mobilities are highly sensitive to detailed fabrication parameters. For example, solution processable FETs made from the most commonly used polymer, regioregular poly(3-hexylthiophene), sensitively depends on the molecular weight, the dielectric-semiconductor interface, the solvent that it is spun from, surface treatments, postfilm formation treatment, and annealing. ${ }^{4-7}$ Furthermore, FETs made from polymers tend to degrade in air, adding another degree of difficulty to the procedure. ${ }^{8}$

An alternative route to fabricate high quality solution processed FETs that can be superior to polymer based devices may be the use of carbon nanotubes (CNTs) dispersed in solution. FETs from individual CNTs have displayed exceptional electrical properties including subthreshold swings as low as $60 \mathrm{mV} /$ decade and mobilities reaching $79000 \mathrm{~cm}^{2} / \mathrm{V} \mathrm{s} .{ }^{9}$ However, devices fabricated from arrays of CNTs can be advantageous over individual tube devices in certain cases, as they may provide more homogeniality from device to device and can cover large areas. In addition, devices fabricated with nanotube arrays contain hundreds of CNTs, which can increase current outputs (up to hundreds of microamperes). Large scale assembly of CNTs from solution can be achieved by several different techniques including chemical and biological patterning, ${ }^{10,11}$ Langmuir-Blodgett assembly, ${ }^{12}$ bubble blown films, ${ }^{13}$ contact printing, ${ }^{14}$ inkjet printing, ${ }^{15}$ spin coating assisted alignment, ${ }^{16}$ and evaporation driven self-assembly. ${ }^{17}$ All of these techniques create CNT networks where charge transport needs to occur through a

\footnotetext{
${ }^{\text {a) }}$ Present address: Chemical Engineering Department, University of Puerto Rico at Mayaguez, Mayaguez 00681-9000, Puerto Rico.

b) Author to whom correspondence should be addressed. Electronic mail: saiful@mail.ucf.edu.
}

large number of overlapping internanotube contacts.

Recently, dielectrophoresis (DEP) has been used for the directed assembly of individual, bundles, or networks of CNTs. ${ }^{18-21}$ However, high quality FETs from large area DEP assembled arrays have not been demonstrated. DEP assembled CNT-FET devices can be advantageous as every CNT connects between source and drain electrodes minimizing charge transport through CNT-CNT interconnects. Here we report on solution processed, large area high quality FETs from dielectrophoreticaly aligned arrays of CNTs. Commercially available, surfactant free CNT solution [suspended in de-ionized(DI) water $]^{22}$ were assembled between source (S) and drain (D) electrodes patterned on a $\mathrm{Si} / \mathrm{SiO}_{2}$ substrate by applying an ac electric field. The highly doped $\mathrm{Si}$ substrate was used as a global back gate (G). After using an electrical breakdown technique to remove the metallic CNT pathways, the devices showed on-off ratios $\left(I_{\text {on }} / I_{\text {off }}\right)$ up to $\sim 2 \times 10^{4}$ with $p$-type FET behavior. The measured mobilities are as high as $123 \mathrm{~cm}^{2} / \mathrm{V} \mathrm{s}$, which is three orders of magnitude higher than typical solution processed organic FETs. Our technique represents a simple and convenient way to fabricate high quality solution processable FET devices.

Highly doped Si wafers with $250 \mathrm{~nm}$ capped layer of $\mathrm{SiO}_{2}$ were used as substrates. Source and drain electrodes with spacing $L=5 \mu \mathrm{m}$ and a width $W=200 \mu \mathrm{m}$ were defined using electron beam lithography (EBL), then electron beam evaporation of $\mathrm{Cr}(5 \mathrm{~nm})$ and $\mathrm{Pd}(30 \mathrm{~nm})$, followed by standard lift-off in acetone. The sample was then placed in oxygen plasma cleaner for $10 \mathrm{~min}$ to remove the unwanted organic residues on the surface.

Surfactant free, highly purified CNTs suspended in DI water were obtained from Brewer Science Inc. ${ }^{22}$ The obtained solution has a concentration of $\sim 50 \mu \mathrm{g} / \mathrm{ml}$. The solution is further diluted in DI water to obtain a concentration of $\sim 1.0 \mu \mathrm{g} / \mathrm{ml}$. The assembly of CNTs was carried out in a probe station under ambient conditions. Figure 1(a) shows a schematic of the DEP assembly circuit. First, a $3 \mu$ drop of the NT suspension was cast onto the electrode array. An ac voltage of $300 \mathrm{kHz}, 5 \mathrm{~V}_{p-p}$ is applied between the source and gate electrode for $15 \mathrm{~s}$. For high frequencies $(f)$ of the ac voltage applied between source and gate, the impedance $(Z$ $\left.=1 / j \omega C_{\text {plate }}, \omega=2 \pi f\right)$ reduces considerably. Therefore the drain becomes capacitively coupled to the gate electrode and 

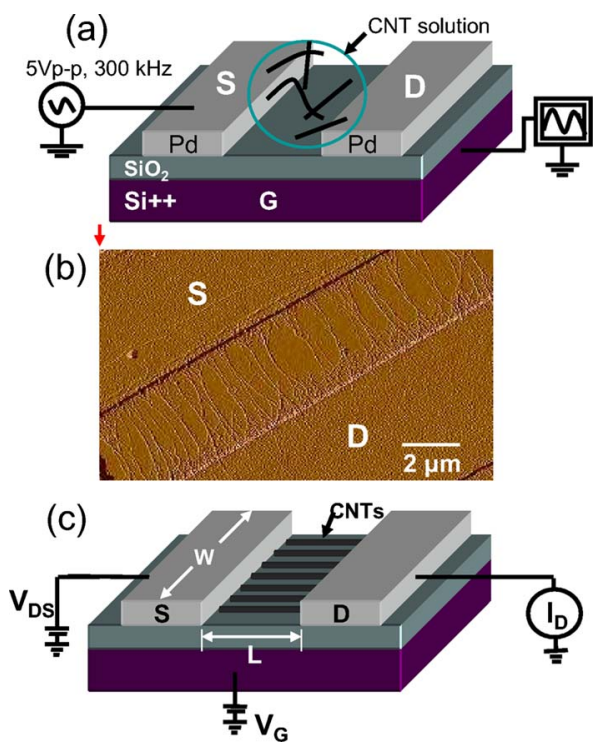

FIG. 1. (Color online) (a) Schematic of dielectrophoretic assembly. An ac voltage of $5 \mathrm{~V}$ at $300 \mathrm{kHz}$ is applied to the source (S) electrode while the conducting Si substrate is monitored by an oscilloscope. (b) AFM image of a section of a device after assembly. (c) Cartoon for electronic transport measurements setup.

obtains a similar potential as the gate electrode creating the necessary potential difference between the source and drain electrodes. Hence, the ac voltage creates a time averaged dielectrophoretic force between source and drain to align the CNTs. For an elongated object, it is given by $F_{\text {DEP }}$ $\propto \varepsilon_{m} \operatorname{Re}\left[K_{f}\right] \nabla E_{\mathrm{rms}}^{2}, K_{f}=\left(\varepsilon_{p}^{*}-\varepsilon_{m}^{*}\right) / \varepsilon_{m}^{*}, \quad \varepsilon_{p, m}^{*}=\varepsilon_{p, m}-i\left(\sigma_{p, m} / \omega\right)$, where $\varepsilon_{p}$ and $\varepsilon_{m}$ are the permittivity of the nanotube and solvent respectively, $K_{f}$ is the Claussius-Mossotti factor, and $\sigma$ is the conductivity. ${ }^{8}$ The induced dipole moment of the nanotube interacting with the strong electric field causes the nanotubes to move in a translational motion along the electric field gradient and align between the source and drain electrodes. As the CNTs assemble between source and drain electrodes, the parallel plate capacitance $\left(C_{\text {plate }}=\varepsilon A / t_{\mathrm{ox}}, t_{\mathrm{ox}}\right.$ is the thickness and $\varepsilon=3.9 \varepsilon_{0}$ is the dielectric constant of the $\mathrm{SiO}_{2}$ layer) of the electrode $/ \mathrm{SiO}_{2} / \mathrm{Si}$ stack increases due to an increase of the effective area $A=W L$. This causes a decrease of the impedance of the drain $/ \mathrm{SiO}_{2} / \mathrm{Si}$ stack. This was evident in the observation of the output signal on the oscilloscope as it increased by $30 \%-40 \%$ by the end of the assembly. Figure 1(b) shows an atomic force microscope (AFM) image for a portion of a device after the assembly. The density of the aligned array is $\sim 1 \mathrm{CNT} / \mu \mathrm{m}$ on average giving $200 \mathrm{CNTs}$ total in the channel. By varying the CNT density of the solution and the trapping time, it is possible to tune the number of CNTs per micron in the array. A detailed study of this assembly will be presented elsewhere. The diameter of the CNTs, measured by AFM varies from 1.5 to $6.0 \mathrm{~nm}$. A total of 16 devices were measured, of which half of the devices are measured as-assembled without further processing (bottom contacted device). The other half was measured following an additional EBL step for which 30 $\mathrm{nm}$ thick Pd was evaporated to form a top contact (top contacted device).

After the assembly, the room temperature electronic transport measurements were carried out in a probe station using the Si substrate as a global back gate. Figure 1(c) shows a schematic of the electrical transport measure-
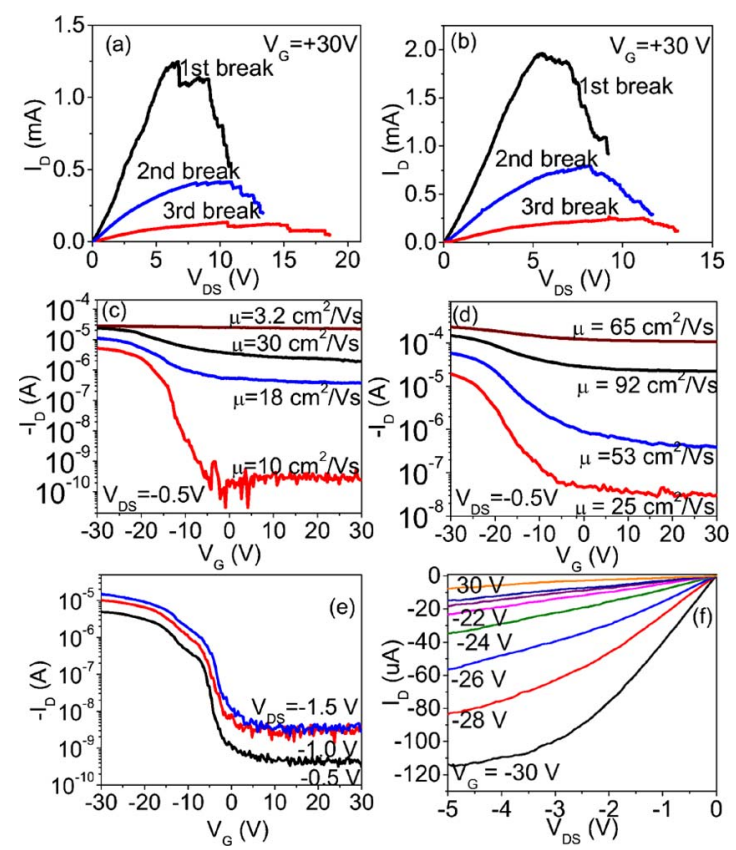

FIG. 2. (Color online) A representative plot of drain current $\left(I_{\mathrm{D}}\right)$ vs sourcedrain voltage $\left(V_{\mathrm{DS}}\right)$ for three sequential breakdowns (first, second, and third break) for (a) a bottom contacted and (b) a top contacted device (c) $I_{\mathrm{D}}$ vs back gate voltage $V_{\mathrm{G}}$ at constant $V_{\mathrm{DS}}$ of $-0.5 \mathrm{~V}$ after the assembly and each breakdown (top to bottom of figure) for the bottom contacted device. (d) $I_{\mathrm{D}}$ vs $V_{\mathrm{G}}$ after assembly and each breakdown (top to bottom of figure) for top contacted device. (e) $I_{\mathrm{D}}$ vs $V_{\mathrm{G}}$ after third breakdown at different $V_{\mathrm{DS}}$ of $-0.5,-1.0$, and $-1.5 \mathrm{~V}$ for the same device as in (c). The on-off ratio for this device is $\sim 2 \times 10^{4}$. (f) Output characteristics for the top contact device presented in (d) after third breakdown.

ment that was performed by means of a Keithley 2400 source-meter, 6517A electrometer, and a current preamplifier interfaced with LABVIEW. The initial two terminal resistance is typically in the range of $20-50 \mathrm{k} \Omega$ for bottom contacted devices and $2-5 \mathrm{k} \Omega$ for the top contacted devices. The mobility is calculated using the formula $\mu$ $=\left(L A / W V_{\mathrm{DS}} C\right) \times\left(d I_{\mathrm{D}} / d V_{\mathrm{G}}\right)$. The capacitance $C$ of the CNT FET array device was approximated from $C$ $=A D /\left\{C_{Q}^{-1}+(1 / 2 \pi \varepsilon) \ln \left[\sinh \left(2 \pi t_{\mathrm{ox}} D\right) / \pi R D\right]\right\}$, where $C_{Q}=4$ $\times 10^{-10} \mathrm{~F} / \mathrm{m}$ is the quantum capacitance, $R$ is the radius of the nanotubes, and $D$ is the linear density in CNTs per micron of the array. ${ }^{23}$ According to this equation, the capacitance increases with increasing density of the nanotube and saturates to parallel plate capacitance value at high enough nanotube density. Here, we used $R=1 \mathrm{~nm}$ and $D$ $=1 \mathrm{CNT} / \mu \mathrm{m}$.

The as-assembled aligned CNT array devices show semimetallic behavior with on-off ratios, $I_{\text {on }} / I_{\text {off }} \sim 1.3-3.0$ and average mobilities of $\mu \sim 5.5$ and $\sim 54 \mathrm{~cm}^{2} / \mathrm{V}$ s for the bottom contact and top contact devices, respectively. The low on-off ratio and modest mobility is due to the presence of large amount of metallic pathways in the array. Therefore to increase device performance, we performed an electrical breakdown procedure to controllably reduce the metallic pathways. $^{24}$

Figures 2(a) and 2(b) show a representative plot of drain current $\left(I_{\mathrm{D}}\right)$ versus source-drain voltage $\left(V_{\mathrm{DS}}\right)$ for three sequential breakdowns (first, second, and third break) for a bottom contacted and a top contacted device, respectively. The back gate was held constant at $V_{\mathrm{G}}=+30 \mathrm{~V}$ to deplete the carriers in the $p$-type semiconducting CNTs while we 

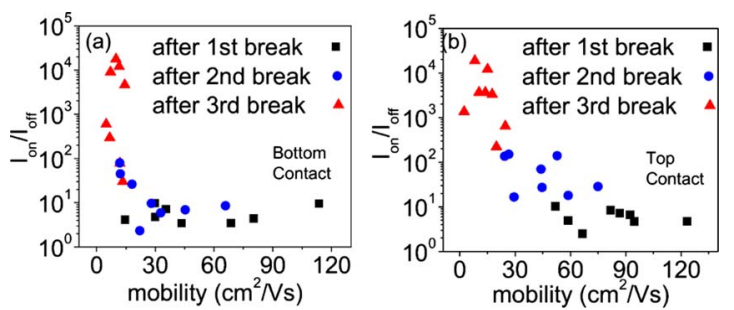

FIG. 3. (Color online) Plot of on-off ratios and corresponding mobility for all measured devices after each breakdown in (a) bottom contact and (b) top contact configuration.

ramped up $V_{\mathrm{DS}}$ to eliminate the metallic CNTs. As $V_{\mathrm{DS}}$ is ramped up, the CNTs start to breakdown and $I_{\mathrm{D}}$ begins to fall. In order to obtain reproducible results, each breakdown is stopped when $I_{\mathrm{D}}$ is about $50 \%$ of its peak value at which point $V_{\mathrm{DS}}$ is swept back to zero. When the third breakdown reaches $\sim 50 \%$ of its peak value, $I_{\mathrm{D}}$ can range from $\sim 0.03$ to $0.12 \mathrm{~mA}$.

Figures 2(c) and 2(d) show $I_{\mathrm{D}}$ versus $V_{\mathrm{G}}$ characteristics for a typical bottom and top contact device, respectively after each breakdown. The source-drain voltage is held constant at $V_{\mathrm{DS}}=-0.5 \mathrm{~V}$. In Fig. 2(c), the uppermost curve is the initial sweep showing a mobility of $3.2 \mathrm{~cm}^{2} / \mathrm{V} \mathrm{s}$ with very little on-off ratio $(\sim 1.1)$. After the first breakdown, the field effect behavior of the device is enhanced; both the mobility and on-off ratios are increased to $30 \mathrm{~cm}^{2} / \mathrm{V} \mathrm{s}$ and $\sim 10$, respectively, due to a reduction of metallic pathways. After the second breakdown, the mobility reduces a small amount to $18 \mathrm{~cm}^{2} / \mathrm{V} \mathrm{s}$ and the on-off ratio increases to $\sim 26$. Finally, after the third breakdown the mobility is reduced to $10 \mathrm{~cm}^{2} / \mathrm{V} \mathrm{s}$; however, the on-off ratio increases a few orders of magnitude to $\sim 2 \times 10^{4}$. Figure $2(\mathrm{~d})$ shows similar behavior for the top contacted device with $\mu=65,92,53$, and $25 \mathrm{~cm}^{2} / \mathrm{V} \mathrm{s}$ and on-off ratios of 2.1, 6.6, 14, and 650 for the initial sample and then after the first, second, and third breakdown, respectively. We find that the top contacted devices show higher mobilities, which most likely is due to the reduced contact resistance. Figure 2(e) is a plot of $I_{\mathrm{D}}$ versus $V_{\mathrm{G}}$ at different $V_{\mathrm{DS}}$ after the third breakdown for the same device shown in Fig. 2(c). Figure 2(f) shows the detailed output characteristics, $I_{\mathrm{D}}$ versus $V_{\mathrm{DS}}$ at different $V_{\mathrm{G}}$ recorded for the sample presented in Fig. 2(d) after the third breakdown.

Figures 3(a) and 3(b) show the on-off ratio and corresponding mobility value for all of the bottom [Fig. 3(a)] and top contact [Fig. 3(b)] devices after each breakdown. In Fig. 3(a) the on-off ratio remains fairly constant and then increases more rapidly after the third breakdown with median on-off ratios after each breakdown of 4.6, 9.2, and 2.6 $\times 10^{3}$. Figure $3(\mathrm{~b})$ shows a more steadily increase in on-off ratio after each breakdown with median values of $5.8,50$, $3.5 \times 10^{3}$. For the bottom contacted devices, the median mobilities are 50,27, and $9.1 \mathrm{~cm}^{2} / \mathrm{V} \mathrm{s}$ after first, second, and third breakdown, respectively. Top contacted devices yield median mobility values of 77,41 , and $15 \mathrm{~cm}^{2} / \mathrm{V} \mathrm{s}$ after the three breakdowns, respectively. We found that the top contacted devices are more controllable and show better device to device reproducibly after each breakdown. This is most likely due to the better contact resistance from the top contact. The highest mobility obtained from all the devices is $123 \mathrm{~cm}^{2} / \mathrm{V} \mathrm{s}$. The mobility values reported here are up to three orders of magnitude higher than typical FET devices made from solution processed polymers. ${ }^{2}$

In conclusion, we have demonstrated solution processable large area FETs from aligned arrays of CNTs. The CNTs were aligned from a commercially available, surfactant free CNTs suspended in aqueous solution using ac DEP. After reducing the metallic pathways using electrical breakdown, the devices displayed on-off ratios up to $\sim 2 \times 10^{4}$. The devices showed $p$-type FET behavior with mobilities up to three orders of magnitude higher than typical solution processed organic FET devices. The ease of processing for the dielectrophoreticly assembled devices presented here offers an alternative to solution processed polymer FET devices.

This work is partially supported by U.S. National Science Foundation under Grant No. ECCS-0748091 (CAREER) and NSF REU Site Grant No. EEC-0453436.

${ }^{1}$ A. Dodabalapur, Mater. Today 9, 24 (2006).

${ }^{2}$ T. B. Singh and N. S. Sariciftci, Annu. Rev. Mater. Res. 36, 199 (2006).

${ }^{3}$ S. K. Park, D. A. Mourey, S. Subramanian, J. E. Anthony, and T. N. Jackson, Appl. Phys. Lett. 93, 043301 (2008).

${ }^{4}$ R. J. Kline, M. D. McGehee, E. N. Kadnikova, J. Liu, and J. M. J. Frechet, Adv. Mater. (Weinheim, Ger.) 15, 1519 (2003).

${ }^{5}$ A. Facchetti, M.-H. Yoon, and T. J. Marks, Adv. Mater. (Weinheim, Ger.) 17, 1705 (2005).

${ }^{6}$ J. F. Chang, B. Q. Sun, D. W. Breiby, M. M. Nielsen, T. I. Solling, M. Giles, I. McCulloch, and H. Sirringhaus, Chem. Mater. 16, 4772 (2004). ${ }^{7}$ L. Ke, S. B. Dolmanan, L. Shen, C. Vijila, S. J. Chua, R.-Q. Png, P.-J. Chia, L.-L. Chua, and P. K.-H. Ho, Appl. Phys. Lett. 93, 153507 (2008).

${ }^{8}$ B. Ong, Y. Wu, L. Jiang, P. Liu, and K. Murti, Synth. Met. 142, 49 (2004).

${ }^{9}$ P. Avouris, Z. H. Chen, and V. Perebeinos, Nat. Nanotechnol. 2, 605 (2007).

${ }^{10}$ S. Auvray, V. Derycke, M. Goffman, A. Filoramo, O. Jost, and J.-P. Bourgoin, Nano Lett. 5, 451 (2005).

${ }^{11}$ K. Keren, R. S. Berman, E. Buchstab, U. Sivan, and E. Braun, Science 302, 1380 (2003).

${ }^{12}$ S. Jin, D. Whang, M. C. McAlpine, R. S. Friedman, Y. Wu, and C. M. Lieber, Nano Lett. 4, 915 (2004).

${ }^{13}$ Y. Guihua, C. Anyuan, and C. M. Lieber, Nat. Nanotechnol. 2, 372 (2007).

${ }^{14}$ A. Javey, S. W. Nam, E. S. Friedman, H. Yan, and C. M. Lieber, Nano Lett. 7, 773 (2007).

${ }^{15}$ K. Kordás, T. Mustonen, G. Tóth, H. Jantunen, M. Lajunen, C. Soldano, S. Talapatra, S. Kar, R. Vajtai, and P. M. Ajayan, Small 2, 1021 (2006).

${ }^{16}$ M. C. LeMieux, M. Roberts, S. Barman, Y. W. Jin, J. M. Kim, and Z. Bao, Science 321, 101 (2008).

${ }^{17}$ M. Engel, J. P. Small, M. Steiner, M. Freitag, A. A. Green, M. C. Hersam, and P. Avouris, ACS Nano 2, 2445 (2008).

${ }^{18}$ P. Stokes and S. I. Khondaker, Nanotechnology 19, 175202 (2008).

${ }^{19}$ A. Vijayaraghavan, S. Blatt, D. Weissenberger, M. Oron-Carl, F. Hennrich, D. Gerthsen, H. Hahn, and R. Krupke, Nano Lett. 7, 1556 (2007).

${ }^{20}$ S. Banerjee, B. White, L. Huang, B. J. Rego, S. O’brien, and I. P. Herman, Appl. Phys. A: Mater. Sci. Process. 86, 415 (2007).

${ }^{21}$ A. H. Monica, S. J. Papadakis, R. Osiander, and M. Paranjape, Nanotechnology 19, 085303 (2008).

${ }^{22}$ See http://www.brewerscience.com for more information about the CNT solution.

${ }^{23}$ S. J. Kang, C. Kocabas, T. Ozel, M. Shim, N. Pimparkar, M. A. Alam, S. V. Rotkin, and J. A. Rogers, Nat. Nanotechnol. 2, 230 (2007).

${ }^{24}$ P. G. Collins, M. S. Arnold, and Ph. Avouris, Science 292, 706 (2001). 